the subject to their final year students. They are still waiting. It is obviously a difficult subject to write about; if it was not, the bookshops would be flooded with volumes. Professor White has had a good try and has been reasonably successful, but in any attempt to condense space physics into 318 pages the omissions always seem more striking than the actual contents. To take one example: in discussing the Earth's atmosphere the author reviews admirably the pressure and density measurements and the associated profiles, but then proceeds to gloss over the vital problem of temperature and the sources of atmospheric heating.

An attempt has been made to condense and unify a large amount of information and summarize and criticize the currently accepted theories. The mathematics is intentionally kept to a minimum and experimental facts, historical discoveries and important ideas are emphasized. The topics that are dealt with in this book are covered very well with excellent summaries of results, theories and measurement techniques and an extensive list of references.

There are chapters on the Earth's radiation belts and the apparatus used to measure these proton and electron concentrations; the Earth's atmosphere, with special reference to pressure, density and composition; the ionosphere, the different ionized regions and ionospheric winds; the Sun, including a study of element abundance, energy transport, magnetic fields and active regions; interplanetary space and the solar wind and, finally, the magnetosphere.

This book will be useful to university students and to research scientists looking for a guide to topics outside their special field, but the high price will drastically curtail the number of people who can benefit from their own individual copy. Surely this is a perfect example of a book which would sell profusely if lower priced.

David W. Hughes

\section{MATHEMATICS OF SPACE}

\section{Space Through the Ages}

The Evolution of Geometrical Ideas from Pythagoras to Hilbert and Einstein. By Cornelius Lanczos. Pp. $x+320$. (Academic Press: London and New York, May 1970.) $80 s ; \$ 11.50$.

This volume can be considered a companion to Professor Lanczos's earlier Disquisition on Fourier Series. Together they attest to his breadth of learning and his expository skill. Although organized and informed by a historical perspective, the book is not a historian's detailed chronicle of problems and solutions in the mathematics of space. Similarly, although much of the text is an exposition of mathematics, it is not a textbook of thoorems and techniques. It is something between, and rather more: the author's personal account of the different fields of mathematics concerned with space, displayed in sequence and expounded lucidly.

The heart of the book is the middle five chapters, from metrical geometry to Einstein's theory of gravitation. Here the author is in complete command of his material, and he takes the reader through the necessary tensor formalism to appreciate the achievements of Gauss, Riemann and Einstein. He shows how their particular style and their commitment to the highest goals conditioned their work; in the case of Einstein, both his successes and his apparent failures.

Readers may find in this book a useful introduction either to the history of metrical geometry or to the mathematics of space and general relativity; but it was written as a personal testament by a cultivated scholar, in an age when vulgarity has penetrated even into mathematics itself.

\section{THANKS TO OROWAN}

\section{Physics of Strength and Plasticity}

Edited by Ali S. Argon. Pp. xxv + 404. (MIT Press: Cambridge, Massachusetts and London, February 1970.) $117 s$.

THrs book is a collection of twenty-seven papers constituting a Fiestschrift presented to Professor Egon Orowan, creator of the theory of crystal dislocations, on his retirement from a chair of mechanical engineering at Massachusetts Institute of Technology. The papers fall into four groups: individual dislocations and basic deformation mechanisms, hardening mechanisms and dislocation dynamics, cracks and fracture, and geology (which is Orowan's most recent enthusiasm). The authors of the papers include many famous names in the theory of dislocation theory, crystal plasticity and fracture, all people who have built in one way or another on Orowan's powerful insights: his papers on the "Orowan stress" in two-phase alloys, on nucleation of twins, on the plastic work term in Griffith's criterion for instability of a crack, are examples much quoted in the book. It seems that Orowan's influence was most pronounced through his lucid, simple and highly stimulating papers, rather than through personal influence, for these papers are almost all by scientists other than his direct collaborators.

The papers are all theoretical in treatment, and many deal in considerable mathematical depth with the implications of a single well-defined model. Orowan's own technique was more impressionistic: he would take a problem, rather than a model, worry it into various attitudes, perform order-of-magnitude calculations to find the weak spot in each model, and thus gradually work his way through to an insight which was usually as simple as it was unexpected. A few of the papers here approximate to this approach. Thus Ashby, who writes on the "Orowan stress", Christian on the nucleation of twins and martensite, Cottrell on the structure of a crack, Kocks on a statistical theory of alloy hardening, and Cohen Vukcevich on a detailed treatment of cleavage initiation in iron by cracking of carbides are papers of this kind. Nabarro pursues a new-found interest in a fascinating discussion of the "disclination structure of insect muscle"--a description of the mutual shear of myosin and actin filaments in terms of the geometrical pattern of their cross-links; this will be of interest to the insect physiologist as well as the adventurous dislocation expert. The latter, however, together with the fracture specialist, are the men to whom this book offers most. Some of the papers here will be quoted for years to come, and a few, Ashby's in particular, may well acquire the status of classics.

R. W. CAHN

\section{FUNCTIONAL ANALYSIS}

\section{Linear Functional Analysis}

Introduction to Lebesgue Integration and Infinite ThresDimonsional Problems. By Bernard Epstein. Pp. $\mathrm{x}+230$. (W. B. Saunders: Philadelphia and London, February 1970.) $81 s$.

Elements of Functional Analysis

By I. J. Maddox. Pp. $x+208$. (Cambridge University Press: London, February 1970.) $50 s ; \$ 6.95$.

Here are two elementary introductory books on functional analysis which could be read by third-year students of mathematics in British universities. The most immediately obvious difference between them lies in their attitude to the Lebesgue integral. More than a third of Epstein's book is devoted to the construction of Lebesgue theory, whereas Maddox makes no essential use of it, preferring, instead, to bring the reader as quickly as possible to functional analysis proper. A second difference concerns the place of linear operators. Maddox treats metric linear spaces and linear functionals on them, but 